\title{
Functional Evaluation of Isolated Zebrafish Hearts
}

\author{
Louise Hecker, ${ }^{1}$ Luda Khait, ${ }^{1}$ Stanley K. Sessions, ${ }^{2}$ and Ravi K. Birla ${ }^{1}$
}

\begin{abstract}
Traditional working heart preparations, based on the original Langendorff setup, are widely used experimental models that have tremendously advanced the cardiovascular field. However, these systems can be deceivingly complex, requiring the maintenance of $\mathrm{pH}$ with $\mathrm{CO}_{2}$, the delivery of oxygenated perfusate, and the need for extensive laboratory equipment. We have examined the feasibility of using isolated zebrafish (Danio rerio) hearts as an experimental model system, in which experimental procedures can be performed in the absence of the traditional requirements and sophisticated setup equipment. Isolated zebrafish hearts exhibited spontaneous contractile activity, could be electrically paced, and were responsive to pharmacologic stimulation with isoproterenol for $1.5 \mathrm{~h}$ after in vivo removal. Isolated zebrafish hearts offer a time- and cost-effective alternative to traditional Langendorff/working heart preparation models, and could be used to investigate cardiac function and repair.
\end{abstract}

\section{Introduction}

$\mathbf{I}_{\mathrm{p}}^{\mathrm{N}}$ N THE United States, cardiovascular disease affects approximately 80 million people and is the leading cause of death. Isolated organ preparations remain a widely used research tool, as they provide an in vitro model independent of systemic in vivo influences. Organ preparations allow a broad spectrum of biochemical, morphological, and pharmacological indices to be measured. The basic requirements for isolated heart preparations include provisions for temperature control, oxygen availability, maintenance of $\mathrm{pH}$, and delivery of perfusate to the heart. Although a wide variety of systems exist, they are largely based on variations of the original Langendorff setup. ${ }^{1,2}$

Langendorff/working heart preparations have tremendously advanced our understanding of cardiovascular physiology; however, these systems have several limitations, can be complex, and may contain several potential pitfalls., ${ }^{2,3}$ Necessary laboratory equipment can be fairly extensive, based on the type of the system used. If the basic requirements (temperature, perfusate, and maintenance of $\mathrm{pH}$ ) are not met, mammalian hearts would cease beating in a matter of seconds or minutes. In addition, all steps, from excision of the organ to initiation of perfusion, are extremely sensitive to time and temperature. Even when extreme precautions are taken during the setup and use of working heart preparations, intrinsic physiological properties of mammalian hearts begin to decline instantly after in vivo removal. For example, spontaneous heart rate in a perfused heart preparation is significantly lower than the physiological norm.
Here, we have investigated the feasibility of using isolated adult zebrafish (Danio rerio) hearts as a model system, where functional, morphological, and pharmacological cardiac tissue properties could be examined.

\section{Materials and Methods}

Culture plates $(35 \mathrm{~mm})$ were prepared as described previously. ${ }^{4,5}$ Briefly, 1.5 mL of polydimethylsiloxane (PDMS) (Dow Chemical, Midland, MI) was poured into each plate and allowed to cure for $24 \mathrm{~h}$. The PDMS substrate provided a layer in which anchor materials could be easily pinned in place. The culture dish was sterilized with $70 \%$ ethanol for $30 \mathrm{~min}$. Ethanol was then aspirated, and culture dishes were exposed to ultraviolet light for $1 \mathrm{~h}$. Zebrafish that were approximately 1 year old were anesthetized by placing them in dechlorinated tap water with $0.5 \%$ MS222 (ethyl 3-aminobenzoate and methanesulfonic acid; Sigma [St. Louis, MO], catalog \#E1052110G) for approximately $1 \mathrm{~min}$. Anesthetized zebrafish were then positioned with their ventral surface facing up under a dissection microscope, and an incision was made through the body wall to expose the heart. Once exposed, the entire heart was excised from the body and placed in a PDMS-coated $35 \mathrm{~mm}$ culture dish with $2 \mathrm{~mL}$ of Lebovitz media (Invitrogen [Carlsbad, CA], catalog \# 11415) supplemented with 20\% fetal bovine serum and $1 \%$ antibiotic-antimycotic. The excised zebrafish hearts remained in the culture dish in modified Lebovitz media for the duration of the experiments.

The protocol for measuring excitability and contractility of isolated zebrafish hearts was adapted from previous work. ${ }^{4,6,7}$

\footnotetext{
${ }^{1}$ Artificial Heart Lab, Division of Cardiac Surgery, University of Michigan, Ann Arbor, Michigan.

${ }^{2}$ Department of Biology, Hartwick College, Oneonta, New York.
} 
FIG. 1. Functional testing setup and histological evaluation. (A) For contractility measurements, the culture dish containing the isolated zebrafish heart was placed on a heated $\left(28^{\circ} \mathrm{C}\right)$ aluminum platform. (B) The apex of the ventricle was anchored to the culture plate, and the other end was attached to the force transducer with minuten pins. (C, D) Staining with Gill's hematoxylin and eosin showed the presence of an intact extracellular matrix with a large number of dispersed cells. The images shown were obtained from a zebrafish heart $1.5 \mathrm{~h}$ after in vivo removal, and photographs were taken using $\times 20$ and $\times 40$ objectives, respectively.
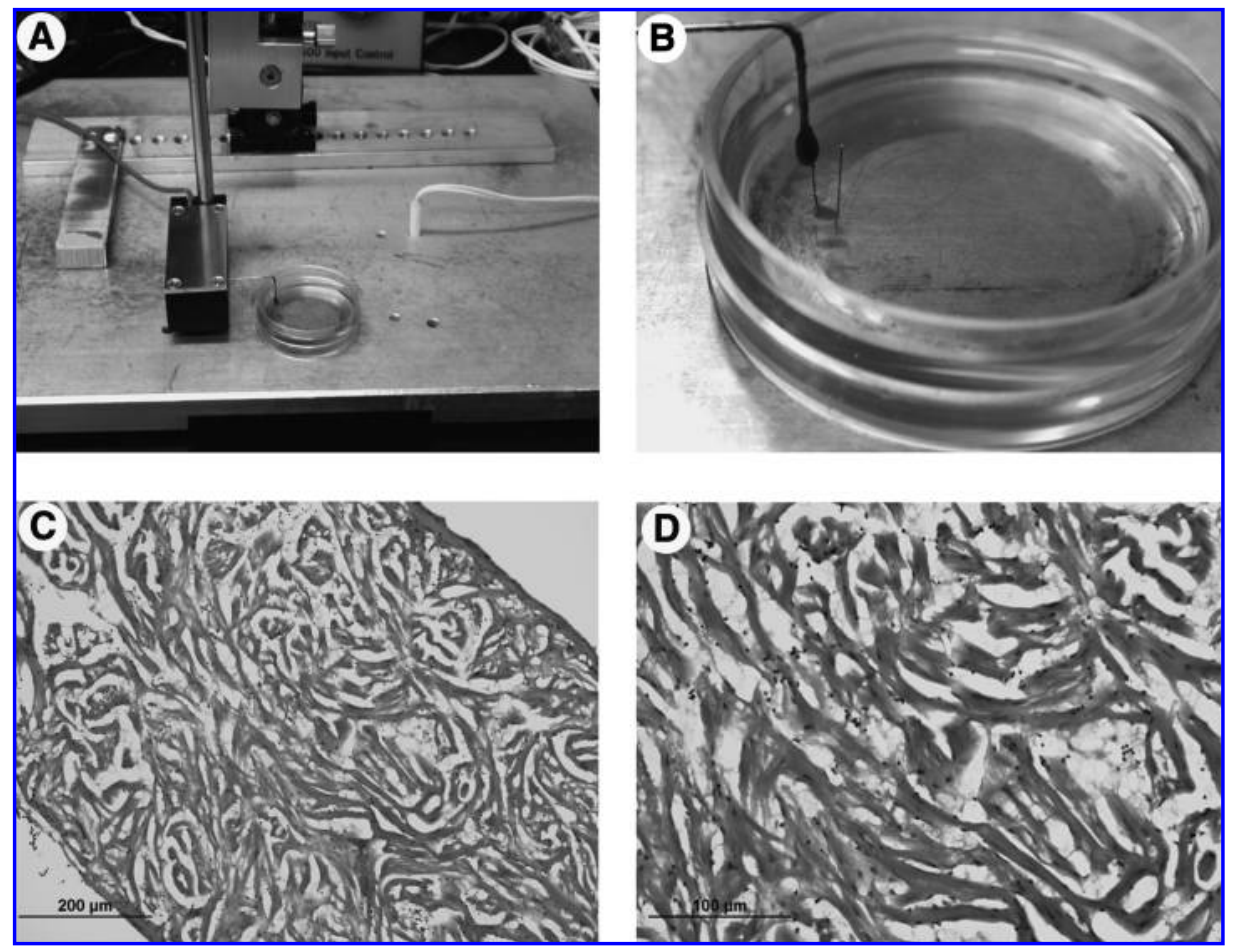

The culture plate was placed on a heated aluminum platform that was maintained at a temperature of $28^{\circ} \mathrm{C}$ (Fig. 1A). The apex of the ventricle was anchored to the PDMS substrate in the culture plate with a $10 \mathrm{~mm}$ by $0.1 \mathrm{~mm}$ diameter stainless steel minuten pin (Australian Entomological Supplies, Bangalow, NSW, Australia). A soldering iron at $150^{\circ} \mathrm{C}$ and canning wax were used to seal a second minuten pin, which was attached to the other end of the heart, to a custom-built optical force transducer (Fig. 1B). The optical force transducer, which has previously been described in detail, ${ }^{4,8}$ has a resolution of $1.4 \mu \mathrm{N}$ and a range of $2 \mathrm{mN}$.

The contractile properties of all zebrafish hearts were evaluated at 0 and $1.5 \mathrm{~h}$ after in vivo removal. Specifically, spontaneous contractility, response to twitch and tetanus electrical stimulus $(10 \mathrm{~V}$, with a frequency of $1 \mathrm{~Hz}$ and a $10 \mathrm{~ms}$ pulse width), and response to isoproterenol were evaluated. Electrical stimulation (twitch and tetanus) was accomplished by placing the heart directly between two parallel platinum electrodes, with a spacing of $5.5 \mathrm{~mm}$. Stimulation was controlled by a computer with Lab-VIEW data acquisition software. The apparatus and protocol for electrical stimulation have previously been described. ${ }^{4,6,8}$ We used isoproterenol at a concentration of $3 \times 10^{-8} \mathrm{M}$, which has previously been demonstrated to elicit contractions in isolated/perfused rat hearts. ${ }^{9}$ After addition of the drug at $0 \mathrm{~h}$, the isolated zebrafish hearts were washed three times with fresh culture media and then remained on the aluminum platform attached to the force transducer throughout testing at $1.5 \mathrm{~h}$. The active force generated by the constructs for all tests was recorded utilizing an optical force transducer, and force tracings were digitally recorded using Lab-VIEW data acquisition software, as previously described. ${ }^{4,6,7}$ Data are presented as means \pm SEM for three to six hearts per data point. Significant differences were determined by ANOVA statistical analysis, and the level of significance was set at $p=0.05$.
After their functional testing, histological evaluation of isolated zebrafish hearts was also performed. Hearts were fixed in $10 \%$ neutral-buffered formalin for approximately $24 \mathrm{~h}$, and prepared for histology as previously described. ${ }^{6}$ Sections were obtained using a cryostat and then were stained with Gill's hematoxylin and eosin to view general structure.

\section{Results}

Isolated zebrafish hearts exhibited spontaneous contractions and were responsive to both twitch and tetanus electrical pacing throughout the duration of all experiments. Representative tracings of electrically stimulated (tetanus and twitch) force generation by isolated zebrafish hearts are shown (Fig. 2A, B). The average twitch force of zebrafish hearts was found to be $258.6 \pm 49 \mu \mathrm{N}$ at the time of isolation $(0 \mathrm{~h})(n=5)$ and $194.3 \pm 60 \mu \mathrm{N}$ at $1.5 \mathrm{~h}$ after isolation $(n=5)$, and there was no significant difference between twitch force generation at 0 and $1.5 \mathrm{~h}$ after isolation (Fig. $2 \mathrm{C}$ ). The average tetanus force of zebrafish hearts was found to be $251 \pm 90 \mu \mathrm{N}$ at $0 \mathrm{~h}$ $(n=3)$ and $170 \pm 32 \mu \mathrm{N}$ at $1.5 \mathrm{~h}$ after removal $(n=3)$ (data not shown).

Isolated zebrafish hearts were also responsive to $3 \times 10^{-8} \mathrm{M}$ isoproterenol (Fig. 3). In response to $3 \times 10^{-8} \mathrm{M}$ isoproterenol, isolated zebrafish hearts exhibited an increase in the number of beats/min and an increase in force generation as compared to controls (with addition of phosphate-buffered saline). Responsiveness of the isolated heart to the drug was similar at both 0 and $1.5 \mathrm{~h}$; therefore, data are expressed as an average of both time points. Addition of the drug increased the heart rate from $51 \pm 7$ to $78 \pm 16$ beats/min $(n=6)$ (Fig. 3B) and increased force generation from $153 \pm 26$ to $385 \pm 144 \mu \mathrm{N}(n=6)$ (Fig. 3C). Therefore, isolated zebrafish hearts exhibited physiological functionality in response to the isoproterenol agonist throughout the duration of the experiment. 

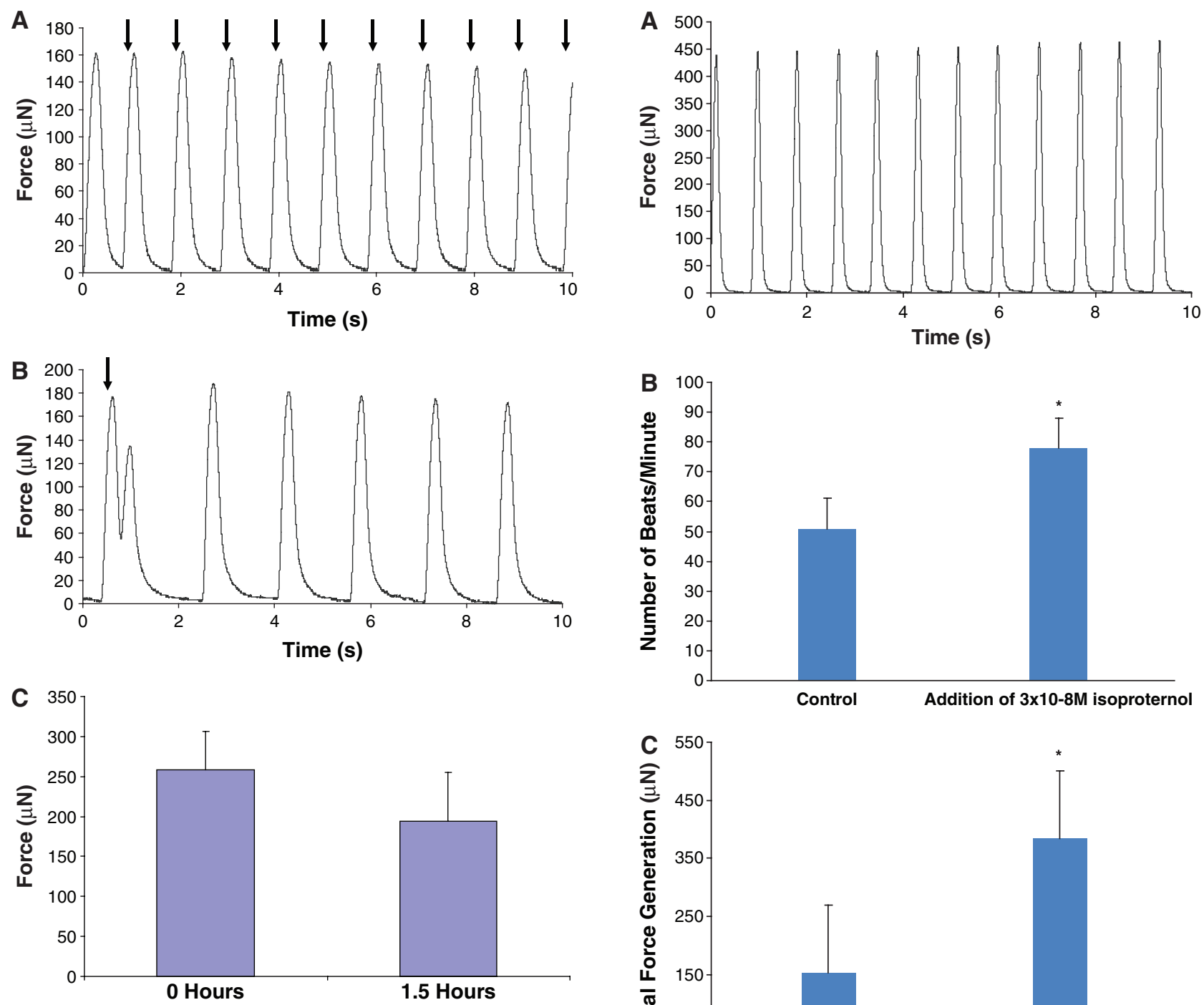

FIG. 2. Spontaneous contractions and electrical pacing. (A) Representative tracing of zebrafish hearts in response to tetanus electrical stimulation. Arrows indicate the onset of electrical stimulation. (B) Representative tracing of zebrafish hearts stimulated with a single twitch electrical impulse, followed by spontaneous contractions. Arrow indicates the onset of electrical stimulation. (C) Graph depicting time course of twitch force generation at 0 and $1.5 \mathrm{~h}$ after in vivo removal, $258.6 \pm 49 \mu \mathrm{N}$ and $194.3 \pm 60$, respectively. Error bars represent standard error of mean $(n=5)$.

Histological evaluation of isolated zebrafish hearts was performed at both 0 and $1.5 \mathrm{~h}$ after in vivo removal $(n=3)$. Histological sections from 0 and $1.5 \mathrm{~h}$ appeared indistinguishable, as no changes in cellular integrity, ruptured cells, or edema could be visually detected. Representative sections of zebrafish hearts from $1.5 \mathrm{~h}$ after isolation were photographed using a Nikon inverted phase contrast microscope, with $\times 20$ and $\times 40$ objectives (respectively) (Fig. 1C, D).

\section{Discussion}

In summary, we have developed a modified working heart preparation using isolated adult zebrafish hearts, which could

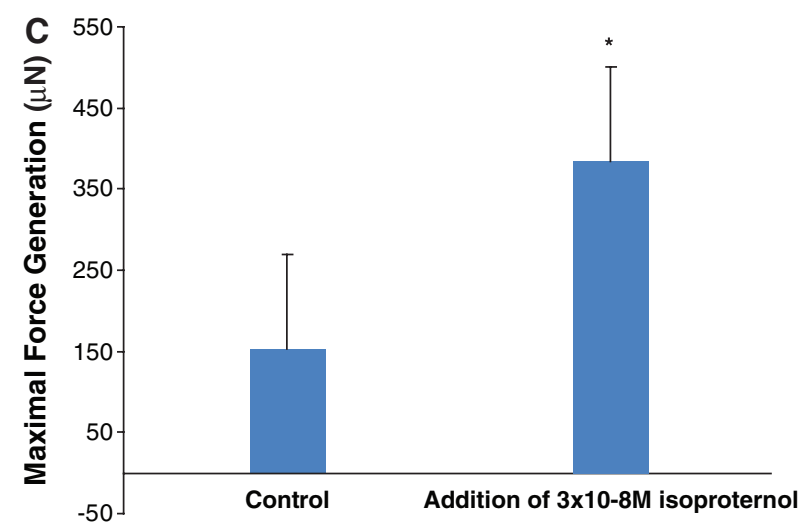

FIG. 3. Response of isolated zebrafish hearts to $3 \times 10^{-8} \mathrm{M}$ isoproterenol. (A) Representative tracing of zebrafish contractile activity in response to $3 \times 10^{-8} \mathrm{M}$ isoproterenol. (B) In response to $3 \times 10^{-8} \mathrm{M}$ isoproterenol, isolated zebrafish heart rate (beats $/ \mathrm{min}$ ) significantly increased from $51 \pm 7$ to $78 \pm 16$ beats/min $(n=6)$. (C) Force generation of isolated zebrafish hearts in response to $3 \times 10^{-8} \mathrm{M}$ isoproterenol significantly increased from $153 \pm 26$ to $385 \pm 144 \mu \mathrm{N} \quad(n=6)$. Error bars represent standard error of mean. ${ }^{*} \mathrm{~A}$ statistical difference, when control (no drug) is compared to the number of beats/min or force generation after addition of the drug $(p<0.05)$.

be used to examine functional, morphological, and pharmacological properties of the heart. The ability of the zebrafish hearts to retain functional contractile properties (spontaneous and electrically stimulated contractions and responsiveness to isoproterenol) for $1.5 \mathrm{~h}$ after in vivo removal provides proof of concept for a powerful, yet simple, tool for functional assessment of cardiovascular function. This in vitro system offers several advantages over currently used methods to study 
cardiac function. First, experimental procedures with isolated zebrafish hearts can be performed in the absence of sophisticated setup equipment/apparatus, such as Langendorff/ working heart preparation apparatus. Attachment of a zebrafish heart to a force transducer can be accomplished in minutes, whereas attachment of an animal heart to Langendorff/ working heart preparation apparatus is time consuming and riddled with potential pitfalls (e.g., damage to the tissue or improper attachment). ${ }^{3}$ Second, using the system described here, perfusion of the heart and $\mathrm{O}_{2} / \mathrm{CO}_{2}$ buffering was not required for maintenance of physiological properties. Lebovitz media, which has previously been used to culture zebrafish cardiomyocytes, ${ }^{10,11}$ contains buffering agents to maintain $\mathrm{pH}$; therefore, $\mathrm{CO}_{2}$ is not required for in vitro cell culture, thus eliminating the need for additional buffering to maintain $\mathrm{pH}$ in the system described here. Traditional Langendorff/ working heart preparations employ perfusion of the heart with oxygenated media to maintain physiological properties. Media perfusion or oxygenation was not necessary to maintain the functional properties of the zebrafish heart, which could be in part due to small size and thickness of the twochambered zebrafish heart in which diffusion may sufficiently deliver oxygen and supplements. Finally, isolated zebrafish heart preparations offer a time- and cost-effective system that could serve as an intermediate step between cell culture and in vivo testing, where high-throughput chemical screening could be evaluated. Contractions of the zebrafish heart could be seen macroscopically, and therefore information, such as the number of beats/min, could be obtained even without a force transducer.

Our data showed no significant decrease in twitch force between the 0 and $1.5 \mathrm{~h}$; however, the average force has decreased at the $1.5 \mathrm{~h}$ time point. Although we statistically evaluated the functional properties of the hearts at 0 and $1.5 \mathrm{~h}$ (Figs. 2 and 3), these isolated hearts exhibited spontaneous contractility for a minimum of $2 \mathrm{~h}$ and could be electrically paced for as long as $5 \mathrm{~h}$ after in vivo removal (data not shown). Future studies are needed to determine a time course of the functional properties of zebrafish hearts after their isolation. In addition, the role of media perfusion on the functional properties of isolated zebrafish also warrants investigation, as active delivery of supplemented media may increase the time period for which these hearts remain functionally viable.

In contrast to mammals, adult zebrafish retain the ability to fully regenerate their heart after injury. After $20 \%$ ventricular amputation, injury-induced cardiomyocyte proliferation occurs and can overcome scar formation, allowing for complete cardiac muscle regeneration in zebrafish. ${ }^{12}$ Biomedical researchers are increasingly exploiting zebrafish for cardiovascular research because they offer several advantages over other model systems. ${ }^{13}$ In the system described here, various injury methods could be used, including focal damage or low oxygen conditions, to investigate cardiac function and/or heart regeneration. Further, genome-wide information for zebrafish and commercially available gene chips enables gene expression profiling and sequence comparison to mammalian cardiac tissue. This model could be used to further elucidate the mechanisms of heart regeneration in zebrafish and could lead to novel regenerative therapies with far-reaching applications to human health.

\section{Disclosure Statement}

No competing financial interests exist.

\section{References}

1. Hearse DJ, Sutherland FJ. Experimental models for the study of cardiovascular function and disease. Pharmacol Res 2000; 41:597-603.

2. Sutherland FJ, Hearse DJ. The isolated blood and perfusion fluid perfused heart. Pharmacol Res 2000;41:613-627.

3. Sutherland FJ, Shattock MJ, Baker KE, Hearse DJ. Mouse isolated perfused heart: characteristics and cautions. $\underline{\text { Clin }}$ Exp Pharmacol Physiol 2003;30:867-878.

4. Huang YC, Khait L, Birla RK. Contractile three-dimensional bioengineered heart muscle for myocardial regeneration. J Biomed Mater Res A 2007;80:719-731.

5. Hecker L, Khait L, Welsh MJ, Birla R. Bioengineering functional human aortic vascular smooth-muscle strips in vitro. Biotechnol Appl Biochem 2008;50:155-163.

6. Hecker L, Khait L, Radnoti D, Birla R. Development of a microperfusion system for the culture of bioengineered heart muscle. ASAIO I 2008;54:284-294.

7. Hecker L, Khait L, Welsh MJ, Birla R. Bioengineering functional human aortic vascular smooth-muscle strips in vitro. Biotechnol Appl Biochem 2008;50:155-163.

8. Dennis RG, Kosnik PE. Excitability and isometric contractile properties of mammalian skeletal muscle constructs engineered in vitro. In Vitro Cell Dev Biol Anim 2000;36:327-335.

9. Tang C, Yang J, Wu LL, Dong LW, Liu MS. Phosphorylation of beta-adrenergic receptor leads to its redistribution in rat heart during sepsis. Am J Physiol 1998;274:R1078-R1086.

10. Baker K, Warren KS, Yellen G, Fishman MC. Defective "pacemaker" current (Ih) in a zebrafish mutant with a slow heart rate. Proc Natl Acad Sci USA 1997;94:4554-4559.

11. Warren KS, Baker K, Fishman MC. The slow mo mutation reduces pacemaker current and heart rate in adult zebrafish. Am J Physiol Heart Circ Physiol 2001;281:H1711-H1719.

12. Poss KD, Wilson LG, Keating MT. Heart regeneration in zebrafish. Science 2002;298:2188-2190.

13. Chico TJ, Ingham PW, Crossman DC. Modeling cardiovascular disease in the zebrafish. Trends Cardiovasc Med 2008; 18:150-155.

Address reprint requests to: Louise Hecker, Ph.D. Artificial Heart Lab Division of Cardiac Surgery University of Michigan 109 Zina Pitcher BSRB Room 2338 Ann Arbor, MI 48109

E-mail: Lhecker@umich.edu 